\title{
COMMISSION 40: RADIO ASTRONOMY (RADIO ASTRONOMIE)
}

\author{
Report of Meetings, 19 and 25 August 1970
}

President: J. P. Wild.

SECRETARY: S. F. Smerd.

\section{Business Meetings}

The President welcomed members of the Commission and the large gathering of non-members present. He recorded with deep regret the death in July 1968 of Professor S. E. Khaikin of Pulkovo Observatory.

The President then reported on two proposals submitted by the Executive Committee for consideration by the Commissions:

(a) Whether the new Organizing Committee should be elected or appointed; the meeting favoured the former.

(b) That the Commission be encouraged to draw up such 'by-laws and guiding principles' as may be useful to future Committees.

The President drew attention to an innovation introduced by the Organizing Committee, namely the preparation of 'Reports on Work in Progress' by radio astronomy observatories; 34 such reports became available for distribution during the Assembly.

The following matters were then discussed;

\section{THE REPORT OF THE COMMISSION}

The President had received overwhelming support for his objection to the apparently arbitrary basis on which the Executive Committee had allocated the maximum length of the Report to each Commission. A resolution had already been submitted to the General Assembly expressing this objection and the General Secretary had foreshadowed the need for new arrangements. The question is to be referred to the incoming Executive Committee.

The Secretary had been notified of one minor amendment; on page 468 , line 3 from the bottom of the page: read (96) for (95).

\section{Membership}

The President reviewed the exchange of suggested deletions and additions between members of the Organizing Committee, the General Secretary and himself which led to the proposed list of Commission- 40 members as submitted to the General Secretary prior to the Assembly. Since then he had, with the agreement of the Organizing Committee, proposed that membership should be open to all IAU-members who were active radio astronomers. This proposal would ensure that a wide and representative body of radio astronomers would receive advance notice of the Commission's programmes at forthcoming meetings. This principle was approved by the meeting. A Subcommittee consisting of Westerhout (convener), Parijskij, Shakeshaft and Smerd was set up to receive nominations. This resulted in the new list of members of Commission 40 as published on page 299 of these Transactions.

\section{INTERFERENCE AND FREQUENCY ALLOCATION}

F. G. Smith reported on the activities of the Inter-Union Commission for the Allocation of Frequencies for Radio Astronomy and Space Science (IUCAF) particularly in preparation for the World Administrative Radio Conference (WARC) under the International Telecommunication 
Union (ITU) in June 1971. Smith has published a summary of the present status of the allocation of frequencies to radio astronomy and of proposed changes in Nature 228, 419, 1970.

It was agreed that two further requirements should be added to this list:

(a) Some protection should be given to observations in the band 235-240 MHz. Radio astronomy was using this band effectively at some locations despite the fact that it is allocated to other services, but proposals now current to allow the use of transmissions from satellites in this band would make it impossible to continue.

(b) Astronomical observations from the far side of the Moon should provisionally be allocated half the electromagnetic spectrum, by concentrating all transmission into selected bands.

\section{ORGANIZATION OF LONG-BASELINE INTERFEROMETRY}

Kellermann suggested that it may be some years before agreement could be reached on the most suitable system and frequency bands for long-baseline interferometry.

\section{COORDINATION OF SOURCE SURVEYS}

Shakeshaft suggested that a Subcommittee of Commission 40 could help in achieving better coordination between radio surveys and between radio and optical surveys; after discussion it seemed agreed that at this stage such coordination may be better achieved through direct cooperation between two or more observatories. However, Shakeshaft and Kellermann were appointed as a Subcommittee to investigate and report on existing surveys and cooperation; M. M. Davis agreed to collate the information and will circulate copies to each observatory.

\section{COORDINATION OF FLUX-DENSITY CALIBRATIONS OF STANDARD SOURCES}

Shakeshaft suggested that Commission 40 should publish flux-density values to an accuracy of, say, $5 \%$ in the range $10 \mathrm{MHz}-10 \mathrm{GHz}$ for a number of standard sources. The Shakeshaft-Kellermann Subcommittee will prepare such a list for the next General Assembly.

\section{FUndaMental positional SYSTEMS For RADio ASTRONOMY}

Ryle pointed out that, with radio astronomy positions now being given to better than $1^{\prime \prime}$ arc, there was a need for a number of calibrators of small angular size; some of their requirements, i.e. appreciable flux density, constancy with time and no low-frequency cut-off, suggest that some sources may be suitable both as position and flux-density calibrators. A Working Group consisting of Ryle (convener), Burke, Christiansen, Cohen, Gent, Palmer and Raimond was constituted to pursue this matter.

\section{TEMPERATURE STANDARDIZATION OF HI SURVEYS}

Van Woerden, the convenor of a Working Group - or Subcommittee - submitted the following report:

"The Subcommittee of Commission 40 on Standardization of $\mathrm{HI}$ surveys recommends that $21-\mathrm{cm}$ line observers compare their results with those of others, and determine the ratio of their brightnesstemperature scales to those of one or more other surveys. For such comparisons, we recommend the standard field $\mathrm{S} 8\left(l^{\mathrm{II}}=207^{\circ}, b^{\mathrm{II}}=-15^{\circ}\right)$, which has been used in several surveys. Some observers have used as standard fields $\mathrm{S} 7\left(l^{11}=132^{\circ}, b^{\mathrm{II}}=-1^{\circ}\right)$ or $\mathrm{S} 9\left(l^{\mathrm{II}}=356^{\circ}, b^{\mathrm{II}}=-4^{\circ}\right)$; we recommend those as secondary standards.

The authors of some of the major surveys are at present intercomparing their scales and expect to publish the results of this in the literature. In view of this, we recommend that the Subcommittee be now dissolved."

The report was adopted and the Subcommittee dissolved. 


\section{THE UNIT OF FLUX DENSITY}

The President suggested that the quantity $10^{-26} \mathrm{~W} \mathrm{~m}^{-2} \mathrm{~Hz}^{-1}$, given a suitable name and symbol, would - in conjunction with the usual decimal prefixes $(\mu, \mathrm{m}, \mathrm{k}, \mathrm{M}$ etc.) - be a desirable unit to express the flux densities throughout the range required in radio astronomy. The proposal lapsed for want of support.

\section{RELATION BETWEEN IAU COMMISSION 40 AND URSI COMMISSION V}

Christiansen pointed out that (a) radio astronomy was in an anomalous position both in IAU and URSI; (b) there was a need to avoid duplication; (c) the IAU had no wish to abolish Commission 40 as long as radio astronomers wanted the latter; (d) URSI was likely to remain a Union for radio science only in spite of UGGI proposals for a merger; and (e) radio astronomers should continue to emphasize techniques at URSI and astronomy at the IAU although there was a clear need for an exchange flow.

\section{NOMENCLATURE FOR SOURCES}

Andrew drew attention to the confusion caused by the use of different nomenclatures in different source surveys; he suggested the general adoption of the Parkes system and pleaded that a source name should not change with improved position determinations.

\section{Contamination OF SPACE}

Lovell suggested that the continued and increased use of space for scientific and other purposes called for a re-affirmation of the appeal to all Governments concerned to consult with the IAU, as expressed in the IAU Resolution No. 1 of 1961. Lovell and Smerd were asked to draft the new resolution: the form in which this was approved by the General Assembly is published as Resolution No. 10 elsewhere in this volume.

Ryle stressed the specific need (see also item 3) for new ITU regulations to ensure that out-of-band transmissions by space vehicles be reduced well below the level currently required for ground-based transmitters. A resolution along these lines as drafted by Hagen and F. G. Smith was approved by the meeting; the form in which the resolution was affirmed by the General Assembly is published as Resolution No. 11 elsewhere in this volume.

\section{ORGANIZATION OF COMMISSION-40 MEETINGS}

Baldwin pointed out that the tight schedule of Commission- 40 meetings made the Chairman's job unpleasant and necessitated overflow meetings. He proposed the following guide-lines to improve future meetings: the three-yearly meetings should not be the place to present (a) 'last week's' work; (b) papers that appeared in publications more than, say, 3 months before the Assembly, and (c) papers also presented at other meetings during the Assembly.

Christiansen drew attention to the Executive's recommendation to limit future Commission meetings to 6 sessions.

Westerhout would be sorry if a re-organization of Commission- 40 meetings prevented young astronomers from presenting their work and becoming known. Lovell drew attention to the need for greater inter-Commission liaison. Following J. A. Robert's complaint that these positive suggestions may be lost, it was agreed to send copies of the minutes to the General Secretary and to members of the new Organizing Committee

\section{ELECTION OF THE ORGANIZING COMMITTEE}

The President presented the proposed list of nominations for the Organizing Committee.

The Commission approved this Committee without further nominations. 


\section{Scientific Meetings}

In addition to the Joint Discussions 'Helium in the Universe', 'Interstellar Molecules', 'Photoelectric Observations of Stellar Occultations' and 'Pulsars and Cosmic Rays' of which accounts are given elsewhere in this volume, Commission 40 had the following scientific meetings:

1. A Joint Meeting organized by Commission 10 on 'Mapping the Sun in X, UV and Radio Wavelengths'; for details see the report of Commission 10.

\section{Extra-Galactic Work}

(with Commission 28) August 20 and 21; organized by J. Lequeux.

P. A. G. Scheuer: 'Extragalactic Radio Sources'.

R. J. Allen: 'Extragalactic Hydrogen Line'.

J. E. Baldwin: 'HI in M 33'.

J. R. Shakeshaft: '5C Survey of the Coma Cluster'.

C. D. Mackay: 'Orientation of the Axes of Radio Galaxies'.

P. P. Kronberg: 'Polarization Measurements'.

H. P. Palmer: 'Structure of Radio Sources'.

P. K. Wraith: 'Structure of Unidentified Radio Sources'.

K. I. Kellermann: 'Very Long Baseline Interferometry'.

J. L. Locke and B. H. Andrew: 'Observation of Variable Sources'.

E. G. Daintree: '11-cm Survey'.

\section{Supernovae Remnants and Pulsars}

(with Commission 34) August 20 and 21; organized by G. Westerhout.

J. A. Roberts: 'Summary of the Crab Nebula Symposium (No. 46) Held at Jodrell Bank'

J. R. Dickel and B. R. Hermann: 'High Resolution Observations of Supernova Remnants'.

M. R. Kundu: 'Polarization Observations of Supernova Remnants at 3 and $6 \mathrm{~cm}$ Wavelengths'.

I. Rosenberg: 'Distribution of Brightness and Polarization in Cassiopeia A at $5.0 \mathrm{GHz}$ '.

D. E. Hogg and R. G. Conway: 'The Polarized Brightness Distribution for the Crab Nebula'.

L. Matveyenko: 'The Brightness Distribution of the Crab Nebula at $3.5 \mathrm{~mm}$ '.

G. S. Downs: 'Interstellar Scintillations in Pulsar Signal at $2388 \mathrm{MHz}$ '.

K. R. Lang: 'Interstellar Scintillation of Pulsar Radiation'.

W. C. Erickson $e t$ al. (read by G. Westerhout): 'VLBI Observations of NP 0531 and Other Small Angular Diameter Radio Sources at $121.6 \mathrm{MHz}$.

M. Cohen: 'VLBI Observations'.

I. S. Shklovsky: 'High-Frequency Flux of the Crab Nebula'.

R. M. Price et al.: 'High Time-Resolution Structure in Pulsars'.

R. Schonhart: 'Polarization and Other Characteristics of NP 0532'.

M. I. Large (read by F. G. Smith): 'Pulsar Luminosity Function'.

J. G. Davies: 'Pulsar Searches'.

T. W. Cole: 'Pulsar Sub Pulses and the Emission Process'.

A. T. Moffet: 'Pulsar Intensities at $3.8 \mathrm{~cm}$ '.

D. C. Backer and F. D. Drake: 'Non-Random Phenomena in Pulsar Pulse Shapes'.

J. M. Rankin: 'Dispersion Measure Change in the Crab Nebula Pulsar'.

C. Heiles: 'Strong Pulses in the Crab Nebula Pulsar'.

M. Grewing and M. Walmsberg: 'On the Interpretation of the Dispersion Measure'.

Y. Terzian: 'Dispersion Measures'.

I. Milogradov-Turin: 'A Survey of the Background Radiation at $38 \mathrm{MHz}$.

E. Berkhuysen: 'Background Survey at $820 \mathrm{MHz}$ '.

R. Wielebinski: 'Background Surveys at 150 and $85 \mathrm{MHz}$ at Parkes, Australia'.

4. Interstellar Radio Spectroscopy (with Commission 34) August 21 and 22; organized by G. Westerhout and Yu. N. Parijskij'. 
H. J. Habing, C. E. Heiles et al.: 'The Hat Creek 21-cm Line Survey at Intermediate and High Latitudes'.

A. Sandqvist and F. J. Kerr: 'Lunar Occultations of the Galactic Center Region in the Hr-, $\mathrm{OH}$ - and $\mathrm{CH}_{2} \mathrm{O}$-lines'.

G. R. Knapp (read by F. J. Kerr): 'High Frequency Resolution Observations of 21-cm Profiles in the Directions of Dense Interstellar Dust Clouds'.

R. Sancisi: 'Neutral Hydrogen in the Region of the Dust Cloud L 134'.

S. J. Goldstein: 'The 21-cm Line Spectrum of the North Galactic Pole'.

M. P. Hughes et al. (read by A. R. Thompson): 'Interferometer Observations of Galactic $21-\mathrm{cm}$ Line Absorption'.

V. Radhakrishnan et al.: '21-cm Absorption Measurements at Parkes'.

F. Sato (read by title only): 'Interstellar Absorption in the Direction of Emission Nebula IC 1795

V. A. Hughes: 'Absorption Measurements in the Direction of W 49'.

K. W. Riegel: '21-cm Absorption Measurements in the Direction of W 31'.

L. E. Snyder and D. Buh1: 'Detection of Emission from an Unidentified Interstellar Molecule'.

B. E. Turner and C. E. Heiles: 'Nonthermal OH Emission from Interstellar Dust Clouds'.

R. D. Davies: 'Mapping of $\mathrm{OH}$ Sources Using Long Baseline Interferometry'.

R. S. Booth: 'OH Variability of VYCMa'.

B. F. Burke et al.: 'Long Baseline Interferometry of $\mathrm{H}_{2} \mathrm{O}$ Line Sources'.

W. T. Sullivan: 'Intensity and Velocity Variations in Galactic Sources of $1.35-\mathrm{cm} \mathrm{H}_{2} \mathrm{O}$ Line Radiation'.

\section{Hn Regions}

(with Commission 34) August 22; organized by G. Westerhout and R. D. Davies.

C. G. Wynn-Williams: 'Maps of the Condensations in W 49A and W3'.

L. A. Higgs: 'A Microwave Survey of Planetary Nebulae'.

Y. Terzian: 'Radio Emission from Small Nebulae'.

R. M. Hjellming and C. M. Wade: 'Radio Emission from Novae'.

R. S. Booth: 'HII Region Interferometry'.

A. Pedlar: 'Stark Broadening in Hil Regions'.

\section{The Sun and Interplanetary Space}

(with Commission 10) 25 August; organized by M. R. Kundu.

J.P. Hagen: (a) 'Observation of a "Smoke Ring" Associated with a Flare'. (b) 'Radio and Optical Observation of 'Two Nearly Simultaneous Limb Flares'. (c) 'Radio and Optical Observation of Flare Showing Mass Motion'.

S. Enome and H. Tanaka (read by T. Takakura): 'Expanding Limb Burst at $3.75 \mathrm{GHz}$ '.

P. Kaufmann: 'Permanent Changes of the State of Polarization after Microwave Bursts'.

H. Urbarz: 'Magnetic Tape Record of Spectral Development of a Narrow Band Type IV Burst'.

J. Roosen: 'Dynamical Spectral Observations of Bursts in the $160-320 \mathrm{MHz}$ Range'.

A. Maxwell: 'New Spectral Observations of Decimetre Bursts'.

G. A. Dulk: 'Radioheliograph Observations of Harmonic Burst Radiation and Implication on Coronal Structure'.

Y. Leblanc: 'Recent Results at Decametre Wavelengths at Nançay and Arecibo'.

R. G. Stone and J. Fainberg: 'RAE Results on Low-Frequency Solar Bursts'.

P. A. Dennison: 'Interplanetary Scintillation and Solar Wind'.

V. V. Vitkevich (read by N. Lotova): 'On Solar Wind Characteristics'.

N. Lotova: 'On the Effective Scale of Irregularities from Scattering and Scintillation Observations'.

R. Ekers: 'Observation of the Acceleration of Solar Wind near the Sun'.

L. T. Little: 'Is the Scale of Interplanetary Medium as Deduced from Interplanetary Scintillations

Compatible with that Derived from Space Probe Observations'.

C. Caroubalos: 'Stereo Project'. 


\section{Radiation Theory and Astrophysical Plasmas}

(with Commission 43) August 21; organized by S. F. Smerd.

V. L. Ginzburg: 'Radiation Mechanisms'.

D. F. Smith: 'Stream Stabilization'.

R. Ramaty: 'Gyro-Synchrotron Radiation and Solar Radio Bursts'.

H. C. Ko: 'Radiation in Magnetoplasma'.

J. P. Wild: 'Simplified Formulae for Synchrotron Radiation'

V. V. Zheleznyakov: 'On the Polarization of Radio Emission from Pulsars'.

V. L. Ginzburg and L. M. Eruchimov: 'The Role of Electron Density Fluctuations in the Determination of the Dispersion and Rotation Measures'.

\section{Fundamental Positional Systems for Radio Astronomy}

(with Commissions 8 and 24) August 21; organized by C. A. Murray and J. R. Shakeshaft.

M. Ryle: 'The Measurement of the Accurate Positions of Radio Sources'.

W. Fricke (read by C. A. Murray): 'A Fundamental System for Planets, Stars, Galaxies and Radio Sources'.

I. I. Shapiro: 'Pulsar Observations and Spherical Astronomy'.

Short contributions were also presented by M. H. Cohen and H. Gent.

9. Planetary and Lunar Radio and Radar Astronomy

(with Commissions 16 and 17) August 24; organized by G. H. Pettengill

\section{Radio Studies}

D. O. Muhleman: 'Interferometer Measurements of Venus and Mercury'.

C. H. Mayer: 'Observations of Venus, Uranus and Neptune'.

A. C. E. Sinclair: 'Interferometer Observations of Venus and Jupiter'.

E. E. Epstein: 'Microwave Spectrum of Mars and 3-mm Data on Mars, Jupiter, Saturn and Uranus'.

A. D. Kuzmin: 'Measurements of Mars Radio Emission at $8.22 \mathrm{~mm}$ and Evaluation of Thermal and Electrical Surface Properties'.

A. D. Kuzmin: 'Measurements of the Radio Emission of Uranus at $8.22 \mathrm{~mm}$ '.

S. Gulkis: 'Jupiter Observations'.

R. G. Conway: 'Polarization of Jupiter's Decimetric Emission'.

G. L. Berge: 'The Position of Jupiter's Radio Emission Centroid at $21 \mathrm{~cm}$ '.

\section{Radar Studies}

G. L. Tyler: 'Bistatic Lunar Observations'.

J. E. B. Ponsonby: 'Mapping the Moon by CW Radar at $162 \mathrm{MHz}$ '.

I. I. Shapiro: 'Topography of the Moon, Mercury and Venus'.

C. C. Counselman: 'Topography of Mars'.

G. H. Pettengill: 'Venus Radar Maps'.

10. Miscellaneous Papers

August 25; organized by M. Ryle.

C. G. T. Haslam: ' $408 \mathrm{MHz}$ Survey'.

J. R. Baker: '408 MHz Polarization'.

R. S. Le Poole: 'Search for Radio Quiet BSO's'.

D. A. Graham and F. G. Smith: 'Pulsar Faraday Rotations'.

J. W. Brooks and J. D. Murray (read by V. Radhakrishnan): 'Zeeman Measurements of Orion A'.

F. D. Drake: 'Performance and Potential of Spherical Reflectors'.

H. D. Greyber: 'Acceleration and Confinement of Plasma Clouds'. 